\title{
Dataset for file fragment classification of video file formats
}

\author{
Narges Sadeghi, Mohadeseh Fahiminia and Mehdi Teimouri
}

\begin{abstract}
Objectives: File fragment classification of video file formats is a topic of interest in network forensics. There are some publicly available datasets for file fragments of various file types such as textual, audio, and image file formats. However, there is no public dataset for file fragments of video file formats. So, in order to evaluate and compare the performance of the classification methods, a challenge is the need to have such datasets.

Data description: In this study, we present a dataset that contains file fragments of 10 video file formats: 3GP, AVI, ASF, FLV, MKV, MOV, MP4, WebM, OGV, and RMVB. Corresponding to each format, the dataset contains the file fragments of video files with different video codec types: H.263, MPEG-4, WMV, H.264, FLV1, H.265, VP8, VP9, Theora, and RealVideo. Totally, 20 different pairs of video format and codec are employed. For each pair of video format and codec, 30,000 file fragments are provided. Totally, the dataset contains 600,000 file fragments.
\end{abstract}

Keywords: Video file formats, Classification, File formats, File fragments

\section{Objective}

A huge part of the Internet traffic is used for transmitting video files. Due to the large size of these files, they are segmented into fragments. The fragments are communicated over the network. Some of these fragments can be received by the network surveillance unit. The network surveillance unit may desire to identify the file format of each fragment for network forensics purposes.

Some researches have been carried in the field of file fragment classification of video file formats [1-3]. There are some publicly available datasets for file fragments of various file formats [4-6]. However, there is no public dataset for file fragments of video file formats. This makes it difficult for other researchers to compare the proposed methods with the existing methods.

In this study, we present a dataset that contains file fragments of 10 video file formats: 3GPP file format (3GP), Audio Video Interleaved (AVI), Advanced systems format (ASF), Flash Video (FLV), Matroska Video

*Correspondence: mehditeimouri@ut.ac.ir

Information Theory and Coding Laboratory, University of Tehran, Tehran, Iran
(MKV), QuickTime Movie (MOV), MPEG-4 Part 14 (MP4), Web Movie (WebM), OGV, and RealMedia Variable Bitrate (RMVB). Corresponding to each format, the dataset contains the file fragments of video files with different video codec types: H.263, MPEG-4, Windows Media Video (WMV), H.264, FLV1, H.265, VP8, VP9, Theora, and RealVideo.

\section{Data description}

First, the whole set of the original video files are prepared. These files are taken from a private archive of Iranian movies and television series. The format of these files is either MKV or MP4. All these videos are encoded by $\mathrm{H} .264$ codec. The frame rate of these videos varies from 15 to 25 frames per second with a frame size from $384 \times 288$ to $1920 \times 1090$. The audio codec of the original video files is either Advanced Audio Coding (AAC) or MPEG Audio Layer-3 (MP3) with bitrates from 128 to 320 kbps.

The set of the original video files are converted in order to obtain video files in 20 different pairs of format/ codec: 3GP/H.263, 3GP/MPEG4, AVI/WMV, AVI/H.264, AVI/MPEG4, ASF/WMV, FLV/FLV1, MKV/H.264, 
Table 1 Overview of data files/data files

\begin{tabular}{|c|c|c|c|}
\hline Label & Name of data file/data file & File types (file extension) & Data repository (DOI) \\
\hline Data file 1 & $01.3 g p-H .263$ & Generic binary data (.dat) & OSF (https://doi.org/10.17605/OSF.IO/8HM5S) \\
\hline Data file 2 & 02. 3gp-MPEG4 & Generic binary data (.dat) & OSF (https://doi.org/10.17605/OSF.IO/8HM5S) \\
\hline Data file 3 & 03. AVI-WMV & Generic binary data (.dat) & OSF (https://doi.org/10.17605/OSF.IO/8HM5S) \\
\hline Data file 4 & 04. AVI-H.264 & Generic binary data (.dat) & OSF (https://doi.org/10.17605/OSF.IO/8HM5S) \\
\hline Data file 5 & 05. AVI - MPEG4 & Generic binary data (.dat) & OSF (https://doi.org/10.17605/OSF.IO/8HM5S) \\
\hline Data file 6 & 06. ASF-WMV & Generic binary data (.dat) & OSF (https://doi.org/10.17605/OSF.IO/8HM5S) \\
\hline Data file 7 & 07. FLV-FLV1 & Generic binary data (.dat) & OSF (https://doi.org/10.17605/OSF.IO/8HM5S) \\
\hline Data file 8 & 08. MKV-H.264 & Generic binary data (.dat) & OSF (https://doi.org/10.17605/OSF.IO/8HM5S) \\
\hline Data file 9 & 09. MKV-H.265 & Generic binary data (.dat) & OSF (https://doi.org/10.17605/OSF.IO/8HM5S) \\
\hline Data file 10 & 10. MKV-MPEG4 & Generic binary data (.dat) & OSF (https://doi.org/10.17605/OSF.IO/8HM5S) \\
\hline Data file 11 & 11. MOV-H.264 & Generic binary data (.dat) & OSF (https://doi.org/10.17605/OSF.IO/8HM5S) \\
\hline Data file 12 & 12. MOV-H.265 & Generic binary data (.dat) & OSF (https://doi.org/10.17605/OSF.IO/8HM5S) \\
\hline Data file 13 & 13. MOV-MPEG4 & Generic binary data (.dat) & OSF (https://doi.org/10.17605/OSF.IO/8HM5S) \\
\hline Data file 14 & 14. MP4-H.264 & Generic binary data (.dat) & OSF (https://doi.org/10.17605/OSF.IO/8HM5S) \\
\hline Data file 15 & 15. MP4-H.265 & Generic binary data (.dat) & OSF (https://doi.org/10.17605/OSF.IO/8HM5S) \\
\hline Data file 16 & 16. MP4-MPEG4 & Generic binary data (.dat) & OSF (https://doi.org/10.17605/OSF.IO/8HM5S) \\
\hline Data file 17 & 17. WebM-VP8 & Generic binary data (.dat) & OSF (https://doi.org/10.17605/OSF.IO/8HM5S) \\
\hline Data file 18 & 18. WebM-VP9 & Generic binary data (.dat) & OSF (https://doi.org/10.17605/OSF.IO/8HM5S) \\
\hline Data file 19 & 19. OGV-Theora & Generic binary data (.dat) & OSF (https://doi.org/10.17605/OSF.IO/8HM5S) \\
\hline Data file 20 & 20. RMVB-RealVideo9-10 & Generic binary data (.dat) & OSF (https://doi.org/10.17605/OSF.IO/8HM5S) \\
\hline Data file 21 & SettingsTable & Portable document format (.pdf) & OSF (https://doi.org/10.17605/OSF.IO/8HM5S) \\
\hline Data file 22 & ConversionSettings & $\begin{array}{l}\text { Archive file format (.zip) containing } 20 \text { port- } \\
\text { able network graphics (.png) files }\end{array}$ & OSF (https://doi.org/10.17605/OSF.IO/8HM5S) \\
\hline Data file 23 & ReadFragments & Matlab script file (.m) & OSF (https://doi.org/10.17605/OSF.IO/8HM5S) \\
\hline
\end{tabular}

MKV/H.265, MKV/MPEG4, MOV/H.264, MOV/H.265, MOV/MPEG4, MP4/H.264, MP4/H.265, MP4/MPEG4, WebM/VP8, WebM/VP9, OGV/Theora, and RMVB/ RealVideo.

Regardless of video format/codec, the contents of any two converted video files are not the same. For each pair of format/codec, we have 60 compressed videos. So, totally we have 1200 video files. Each of these files is segmented into 1 Kbyte (i.e. 1024 bytes) fragments. Then, 500 fragments are randomly selected among the fragments of each file. Same as [6], before randomly selecting the fragments, $12.5 \%$ of the initial fragments and $12.5 \%$ of the final fragments of each file are discarded.

For each pair of video format/codec, we have 30,000 file fragments. So, the dataset of file fragments contains 600,000 file fragments. The dataset is partitioned according to 20 different pairs of video format/codec. Each partition is represented by an individual data file shown in Table 1. For example, data file 1 (i.e. 01. 3gp-H.263.dat) contains 30,000 fragments of 3GP/H.263 files. Data files are provided in a generic binary data file format with.dat file extension.

Data file 21 (i.e. SettingsTable.pdf) contains a table, in which, the software program employed for generating the videos of each pair of format/codec is specified. In this table, the employed compression settings are also specified. Data file 22 (i.e. ConversionSettings.zip) contains several screenshots of the software programs that display the employed compression settings. These screenshots actually demonstrate how the videos are converted. Data file 23 (i.e. ReadFragments.m) is a script in MATLAB language that reads all the fragments from one or more data files. The details of this script are given in [6].

\section{Limitations}

- The size of the fragments is considered to be fixed and equal to 1024 bytes.

- A defined subset of video formats and codecs are considered.

- The number of raw data files for each pair of video format and codec is 60 .

Abbreviations

3GP: 3GPP file format; AAC: Advanced Audio Coding; ASF: Advanced systems format; AVI: Audio Video Interleaved; FLV: Flash Video; MKV: Matroska Video; 
MOV: QuickTime Movie; MP3: MPEG Audio Layer-3; MP4: MPEG-4 Part 14; RMVB: RealMedia Variable Bitrate; WebM: Web Movie; WMV: Windows Media Video.

\section{Acknowledgements}

Not applicable.

\section{Authors' contributions}

MT designed the study. MF and NS collected the data. MT, MF, and NS wrote the code. MT wrote the original draft of the manuscript. All authors read and approved the final manuscript.

\section{Funding}

The authors declare no source of funding.

\section{Availability of data materials}

The data described in this Data note can be freely and openly accessed on OSF at https://doi.org/10.17605/OSF.IO/8HM5S [7]. Please see Table 1 and reference list for details and links to the data.

\section{Ethics approval and consent to participate}

No human subjects were part of this study and permission was thus not required according to the Institutional Review Board guidelines of author one.

\section{Consent for publication}

Not applicable.

\section{Competing interests}

The authors declare that they have no competing interests.
Received: 28 December 2019 Accepted: 20 March 2020

Published online: 15 April 2020

\section{References}

1. Jin X, Kim J. Video fragment format classification using optimized discriminative subspace clustering. Signal Process Image Commun. 2016:40:26-35.

2. Jin X, Kim J. A practical video fragment identification system. Int J Multimedia Ubiquitous Eng. 2015;10(6):165-76.

3. Gloe T, Fischer A, Kirchner M. Forensic analysis of video file formats. Digit Invest. 2014:11:S68-76.

4. Fakouri $R$, Teimouri M. Dataset for file fragment classification of image file formats. BMC Res Notes. 2019;12:774. https://doi.org/10.1186/s1310 4-019-4812-0.

5. Mansouri Hanis F, Teimouri M. Dataset for file fragment classification of textual file formats. BMC Res Notes. 2019;12:801. https://doi.org/10.1186/ s13104-019-4837-4

6. Khodadadi A, Teimouri M. Dataset for file fragment classification of audio file formats. BMC Res Notes. 2019;12:819. https://doi.org/10.1186/s1310 4-019-4856-1.

7. Sadeghi N, Fahiminia M, Teimouri M. Video file fragments dataset and code. OSF. 2019. https://doi.org/10.17605/OSF.IO/8HM5S.

\section{Publisher's Note}

Springer Nature remains neutral with regard to jurisdictional claims in published maps and institutional affiliations.
Ready to submit your research? Choose BMC and benefit from:

- fast, convenient online submission

- thorough peer review by experienced researchers in your field

- rapid publication on acceptance

- support for research data, including large and complex data types

- gold Open Access which fosters wider collaboration and increased citations

- maximum visibility for your research: over 100M website views per year

At BMC, research is always in progress.

Learn more biomedcentral.com/submissions 